\title{
Neoadjuvant Chemotherapy and Radical Surgery in Locally Advanced Cervical Cancer During Pregnancy: Case Report and Review of Literature
}

\author{
Zohreh Yousefi, Azam Hoseini Hoshyar, Sima Kadkhodayan, Maliheh Hasanzade, \\ Mahmoud reza Kalantari, Mansoureh Mottaghi
}

Received: 26 Oct 2012 / Accepted: 22 Dec 2012

(C) OMSB, 2013

\section{Abstract}

For pregnant patients with cervical cancer, treatment recommendations are individualized and dependent on the stage of the disease, gestational age at the time of diagnosis, and the patient's desire as to the cosntinuation of the pregnancy. The aim of this study is to describe the outcome of neoadjuvant chemotherapy with radical surgery and pelvic lymphadenectomy in a woman with cervical cancer who wished to maintain her pregnancy. This is a report of a 26week pregnant woman with locally advanced cervical cancer stage $\mathrm{I}_{\mathrm{b} 2}$ (FIGO) who was successfully treated with neoadjuvant chemotherapy Paclitaxel plus platinum, followed by $\mathrm{C} / \mathrm{S}$ and radical surgery. Her neonate was healthy and had no abnormalities. This case was the first cervical cancer during pregnancy that was treated using this method at the tumor clinic, Mashhad University of Medical Sciences, Iran. Neoadjuvant chemotherapy is an effort to allow time for the fetal to reach viability by preventing the progression of the disease.

Keywords: Locally advanced cervical cancer; Neoadjuvant chemotherapy; Pregnancy; Radical surgery.

\section{Introduction}

$\mathrm{T}$ he incidence of cancer during pregnancy is unclear, but is estimated to be around 1 in 10,000 individuals. After breast and hematological cancers, cervical cancer is one of the most commonly encountered malignancies during pregnancy. ${ }^{1}$ After the diagnosis of cancer during pregnancy, a multidisciplinary decision with a focus on the medical and psychological aspects and fetal viability is needed. Therapeutic management of cancer in pregnancy is a critically difficult choice, especially because the fate of the unborn child is being decided on.

Zohreh Yousefi, Azam Hoseini Hoshyar, Sima Kadkhodayan, Maliheh Hasanzade, Mansoureh Mottaghi $₫$

Department of Obstetrics and Gynecology, Ghaem Hospital School of Medicine, Mashhad University of Medical Sciences, Mashhad, Iran.

E-mail: mmotaghi36@gmail.com

Mahmoud reza Kalantari

Department of Pathology, Ghaem Hospital School of Medicine, Mashhad University of Medical Sciences, Mashhad, Iran.
Gestational age of the pregnancy is a critical issue. Avoiding unnecessary delay that causes risk to the unborn fetus is considered an optimal therapy. Each case is unique and the therapy must be carefully individualized. The diagnosis of invasive cervical cancer during the third trimester of pregnancy may become a valuable option with an effort to allow time to reach fetal viability by preventing progression of the disease, by keeping the fact that a modest delay of therapy has not been shown to impact negatively on maternal and fetal survival in mind. ${ }^{2}$ Fetal evaluation should be managed after 28 weeks with a serial obstetrical ultrasonography, and nonstress testing, biophysical profile testing, as well as umbilical artery Doppler evaluation. ${ }^{3}$ If the patient desires to postpone the therapy to optimize fetal outcome, neoadjuvant chemotherapy (NACT) may then be considered.

NACT may become a valuable option in an effort to allow time to reach a fetal viability by preventing the progression of the disease. It can be considered as a "plan B" in selected women with locally advanced cervical carcinoma who do not want to terminate their pregnancy; however, potential toxicity for the developing fetus must be considered. ${ }^{4}$ Multiple reports exist about good prognosis in mother and child with advisable delay in therapy to permit gestational advancement. Duggan et al. reported a treatment interval of 144 days in eight pregnant patients with FIGO I and $\mathrm{I}_{b}$ cervical cancer who postponed therapy to optimize fetal outcome. ${ }^{5}$ Platinum-based NACT was generally well tolerated. KyoungChul Chun reported chemotherapy with Paclitaxel plus Platinum followed by radical surgery in early stage of cervical cancer during pregnancy in three cases. ${ }^{1}$

Additional reports exist that describe patients who were managed with Cisplatin and Vincristine for four cycles between 23 to 33 weeks of pregnancy with a good long-term outcome. ${ }^{3}$ However, NACT followed by radical surgery in a bulky stage $I_{b 1}$ cervical cancer has been reported as an unsuccessful treatment. ${ }^{6}$

The aim of this report is to review this subject and highlight the merits of using neoadjuvant chemotherapy. We report our first NACT case of a pregnant patient with locally advanced cervical carcinoma; the disease was classified as FIGO Stage $\mathrm{I}_{\mathrm{b} 2}$ which was successfully treated with neoadjuvant chemotherapy, followed by radical surgery. 


\section{Case Report}

A 37 year-old woman gravid- 4, Para- 3, with a history of vaginal bleeding before her pregnancy, referred to the division of tumor clinic of gynecological oncology at Mashhad University of Medical sciences at 26 weeks of gestation. She had no history of medical or surgical diseases; all of her three deliveries were vaginal, and she had not taken a pap test during the past ten years. In pelvic examination, we noted a large friable polypoid lesion protruding from the cervix. The tumor was approximately $5 \mathrm{~cm}$ in diameter, and there was no involvement of the parametrial tissues. Biopsy of the lesion was proved as squamous carcinoma of the cervix (Fig. 1). This diagnosis of cervical cancer as FIGO stage $\mathrm{I}_{\mathrm{b} 2}$ led us to decide to perform surgery regardless of the fetus. This decision was due to the unsuitability of our NICU for the presence of a preterm baby, and permission for 8 more weeks until gestational reached at least 34 weeks was a risk to be taken, but sessions, she accepted to undergo NACT and permit gestational advancement. She subsequently received chemotherapy with paclitaxel $60 \mathrm{mg} / \mathrm{m}^{2}$ and Cisplatin 80 $\mathrm{mg} / \mathrm{m}^{2}$. The treatment cycle was repeated after 10 days, a total of 4 cycles. The patient had no significant hematological, renal or liver toxicity, she only suffered from nausea and vomiting.

The fetus was followed with serial ultrasonography and did not demonstrate intrauterine growth restriction. Evaluation of tumor response during neoadjuvant chemotherapy was through vaginal examination by three expert gynecologist oncologists who found tumor regression. The patient was advised to undergo definitive treatment immediately after she received final chemotherapy. We decided to perform elective cesarean hysterectomy three weeks after the final chemotherapy. One week after 4 cycles of NACT, when the lab test was not suitable for major surgery, her active labor was begun and obligatory emerging classic cesarean section was performed because of the invasive cervical cancer. She gave birth to a 32-33 week baby boy; the newborn was $2800 \mathrm{~g} ; 40 \mathrm{~cm}$; its Apgar scores 8/10 and without any minor or major anomaly. After two weeks, she underwent radical type three hysterectomy accompanied with pelvic and Para eortic lymphadenectomy and both ovarian transpositions.

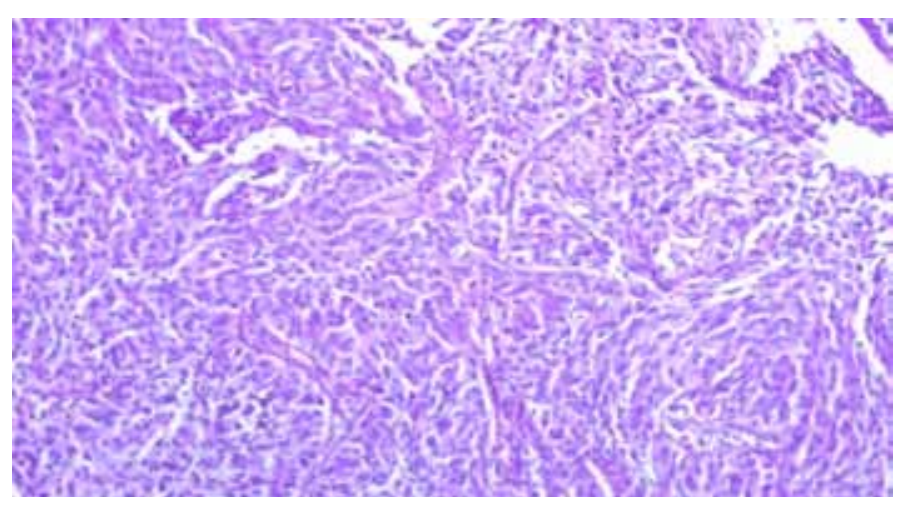

Figure 1: invasive large cell non keratinizing scc. The figure show sheat of neoplastic squmous cells with cytonuclear atypia and pleomorphis without keratinization (H\&E staining, $\times 100)$.
Pathologic report showed micro invasive carcinoma without any cancer in other tissues (Fig. 2), so we encountered a very good response by using the NACT method. Because the stage was $I_{b 2}$ and she was our first NACT case of pregnancy with locally advanced cervical carcinoma, further adjuvant radiotherapy was proposed. Pediatric follow-up of the newborn showed no signs of any metabolic or hematologic abnormality. In the last follow-up (6 months post-surgery), both the mother and infant were in generally good condition.

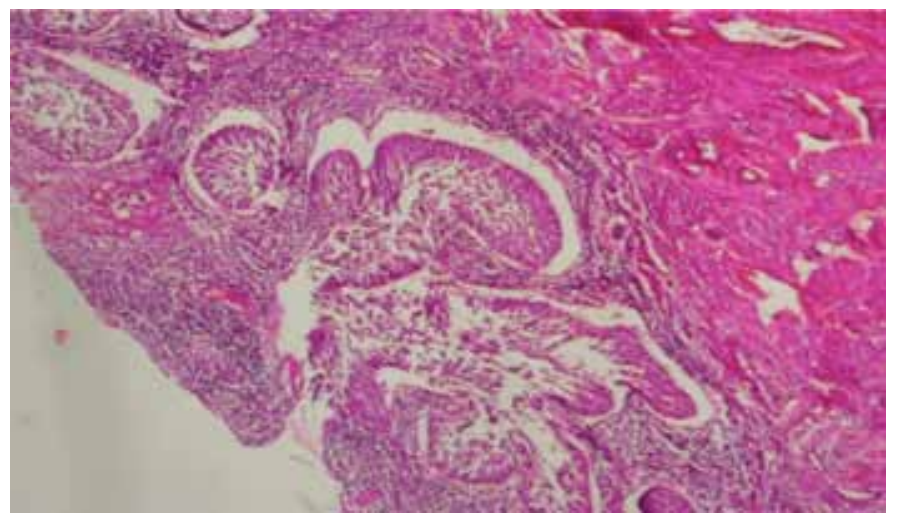

Figure 2: Low power view of micro invasive squmous cell carcinoma of cervix. The figure reveals nests of S.C.C with clefts-like retraction space (H\&E staining, $\times 40)$.

\section{Discussion}

Optimal management of patients with primary tumors greater than $4 \mathrm{~cm}$ in diameter (Stage $\mathrm{I}_{\mathrm{b} 2}$ ) cervical Carcinoma is controversial. Treatment options include: 1) radiation therapy with sensitizing chemotherapy; 2) radical hysterectomy; and 3) NACT with subsequent radical hysterectomy, followed by possible postoperative radiation therapy. Both radical surgery and radiation therapy are equally effective?

This case report constitutes the first description of the use of NACT for invasive carcinoma of the cervix in pregnancy at our center. Ideally, cancer and the pregnancy must be managed without negatively affecting outcomes for neither the baby nor the mother. This is not always possible and we can face dilemmas regarding therapy and the fate of the unborn child; however, appropriate management in these situations is absolutely essential. NACT in an effort to allow time for fetal viability is attainable by preventing progression of disease. The advantage of NACT includes significant reduction of the bulk of cervical tumor and the increase of operability of the lesion. NACT has been proved effective in decreasing the incidence of lymph node metastases and making radical hysterectomy at the time of Cesarean section feasible. A better vascularity allows a higher concentration of drug to be delivered to the tumor bed before pelvic surgery. It is likely that NACT improves survival rate and may reduce the recurrence of the disease. However, lesions that receive delayed treatment have not been shown to impact negatively on maternal survival. And generally, a delay of therapy (maximum one month for continuation vs, termination of pregnancy is without negatively affecting outcomes for the baby or the mother. Several 
NACT agents have been administered to patients with cervical cancer, making it difficult to draw definite conclusion. However, the patients treated with Paclitaxel $200 \mathrm{mg} / \mathrm{m}^{2}$ and Cisplatin $100 \mathrm{mg} /$ $\mathrm{m}^{2}$ which was repeated after 3 weeks of treatment cycle, tolerated the medication well. ${ }^{8}$ Neoadjuvant chemotherapy with Cisplatin, Bleomycin, and Vinblastine have been the most extensively used drug combination. We treated the current patient with $60 \mathrm{mg} / \mathrm{m}^{2}$ Taxol and $80 \mathrm{mg} / \mathrm{m}^{2}$ Cisplatin every 10 days for four cycles after normalization lab tests, and no complication either for mother or her baby was traced. Based on the pathological report, we observed very good pathological response and our patent was successfully treated with chemotherapy and there was no need for post-operative radiotherapy, but because of the possibility of late recurrences, we decided to add a post-operative radio therapy according to NACT in esophageal cancer. Complete clinical response rates with NACT were reported, ranging from $17 \%$ to $44 \%$, and overall response rates were $80 \%$ to $90 \%{ }^{9}$ Difficulty in therapy in patients who do not respond to NACT must be considered; in this situation, curative treatment may become a valuable option. ${ }^{10}$ We must pay special attention to the radio-resistant clones, and the possibility of crossresistance between chemotherapeutic drugs and radiotherapy. ${ }^{5}$

\section{Conclusion}

This therapeutic option should be considered in selected women with locally advanced cervical carcinoma who do not want to terminate their pregnancy.

\section{Acknowledgements}

The authors reported no conflict of interest and no funding was received for this work.

\section{References}

1. Chun KC, Kim DY, Kim JH, Kim YM, Kim YT, Nam JH. Neoadjuvant chemotherapy with paclitaxel plus platinum followed by radical surgery in early cervical cancer during pregnancy: three case reports. Jpn J Clin Oncol 2010 Jul;40(7):694-698.

2. Palaia I, Pernice M, Graziano M, Bellati F, Panici PB. Neoadjuvant chemotherapy plus radical surgery in locally advanced cervical cancer during pregnancy: a case report. Am J Obstet Gynecol 2007 Oct;197(4):e5-e6.

3. da Fonseca AJ, Dalla-Benetta AC, Ferreira LP, Martins CR, Lins CD. [Neoadjuvant chemotherapy followed by radical surgery in pregnant patient with invasive cervical cancer: case report and literature review]. Rev Bras Ginecol Obstet 2011 Jan;33(1):43-48.

4. Boyd A, Cowie V, Gourley C. The use of cisplatin to treat advanced-stage cervical cancer during pregnancy allows fetal development and prevents cancer progression: report of a case and review of the literature. Int J Gynecol Cancer 2009 Feb;19(2):273-276.

5. Duggan B, Muderspach LI, Roman LD, Curtin JP, d'Ablaing G III, Morrow CP. Cervical cancer in pregnancy: reporting on planned delay in therapy. Obstet Gynecol 1993 Oct;82(4 Pt 1):598-602.

6. Gottschalk E, Mangler M, Schneider A, Koehler C, Lanowska M. Pregnancy after lymphadenectomy and neoadjuvant chemotherapy followed by radical vaginal trachelectomy in FIGO stage IB1 cervical cancer. Fertil Steril 2011 Jun;95(7):e5-e7.

7. Li J, Wang LJ, Zhang BZ, Peng YP, Lin ZQ. Neoadjuvant chemotherapy with paclitaxel plus platinum for invasive cervical cancer in pregnancy: two case report and literature review. Arch Gynecol Obstet 2011 Sep;284(3):779-783.

8. Marchiole P, Tigaud JD, Costantini S, Mammoliti S, Buenerd A, Moran E, et al. Neoadjuvant chemotherapy and vaginal radical trachelectomy for fertilitysparing treatment in women affected by cervical cancer (FIGO stage IB-IIA1). Gynecol Oncol 2011 Sep;122(3):484-490.

9. Rabaiotti E, Sigismondi C, Montoli S, Mangili G, Candiani M, Viganò R. Management of locally advanced cervical cancer in pregnancy: a case report. Tumori 2010 Jul-Aug;96(4):623-626.

10. Islam S, Mukhopadhyay L, Howells R. Neo-adjuvant chemotherapy and radical surgery for stage 1B cervical cancer in pregnancy. J Obstet Gynaecol 2012 Feb;32(2):191-192. 